\title{
Determination of Surface Roughness in Wire and Arc Additive Manufacturing Based on Laser Vision Sensing
}

\author{
Jun Xiong ${ }^{*}$, Yan-Jiang Li, Zi-Qiu Yin and Hui Chen
}

\begin{abstract}
Wire and arc additive manufacturing (WAAM) shows a great promise for fabricating fully dense metal parts by means of melting materials in layers using a welding heat source. However, due to a large layer height produced in WAAM, an unsatisfactory surface roughness of parts processed by this technology has been a key issue. A methodology based on laser vision sensing is proposed to quantitatively calculate the surface roughness of parts deposited by WAAM. Calibrations for a camera and a laser plane of the optical system are presented. The reconstruction precision of the laser vision system is verified by a standard workpiece. Additionally, this determination approach is utilized to calculate the surface roughness of a multi-layer single-pass thin-walled part. The results indicate that the optical measurement approach based on the laser vision sensing is a simple and effective way to characterize the surface roughness of parts deposited by WAAM. The maximum absolute error is less than $0.15 \mathrm{~mm}$. The proposed research provides the foundation for surface roughness optimization with different process parameters.
\end{abstract}

Keywords: Wire and arc additive manufacturing, Surface roughness measurement, Laser vision sensing,

Three-dimensional reconstruction

\section{Introduction}

As opposed to traditional subtractive manufacturing process, additive manufacturing is an advanced technology for building complicated parts directly from a threedimensional (3D) model, by depositing materials in the form of powder or wire [1]. With an increasing emphasis on sustainability, this technology offers great advantages of decreasing buy-to-fly ratios, especially for thin-walled parts. In recent years, research efforts have been devoted to fabricating metal parts with a view to minimizing materials waste, particularly for metals which are either expensive to purchase or hard to machine.

Aiming at a higher deposition rate and a lower cost, wire and arc additive manufacturing (WAAM) has been demonstrated to be a powerful process for fabricating metallic parts. Using arc as the heat source can greatly decrease the equipment cost, for example, gas tungsten

*Correspondence: changfeng0007@163.com

School of Materials Science and Engineering, Southwest Jiaotong University, Chengdu 610031, China arc welding (GTAW) [2-4], plasma arc welding (PAW) [5, 6], and gas metal arc welding (GMAW) [7-12]. Deposition in the form of wire offers significant merits over using powder as the adding material in terms of material waste and contamination. This is because all the wire is fed into the molten pool [13], whereas powder-based techniques have to recycle powders which may produce contamination.

Publications in WAAM are mainly focused on several aspects, such as forming technology [2, 3, 6-8, 12], layer geometry optimization $[10,11]$, sensing and control of layer geometry $[14,15]$, microstructure and mechanical property $[2,6]$, evolution of temperature distribution and residual stress $[16,17]$, etc. For instance, the process window in multi-layer single-pass plasma wire deposition for Ti-6Al-4V was obtained [2]. To keep layer geometry consistent, a vision-sensing and closed-loop control system was developed to increase the process stability [15]. Microstructure and tensile strength of Ti-6Al-4V components deposited by GTAW were presented [1]. Residual 
stresses of parts were reduced in GMAW-based additive manufacturing through high-pressure rolling [17].

Nevertheless, due to the large layer height produced in multi-layer deposition during WAAM process, an obvious stair step occurs between two layers. This effect will greatly deteriorate the surface quality of fabricated parts. Compared to the powder-based additive manufacturing, the surface quality of the part deposited by melting wire is inferior. To meet general industrial requirements [18], the surface quality improvement in WAAM has been a critical challenge. For a better understanding of the dependence of surface quality on process parameters, a methodology for quantitatively characterizing the surface quality in WAAM has to be developed in advance. To our knowledge there are few papers related to quantification of surface roughness in WAAM. By contrast, several studies have been focused on this subject for fused deposition modeling $[19,20]$ and laminated object manufacturing [21, 22]. Most of previous studies are based on theoretical models with a certain assumption. Calculated values are reasonable in limited regions.

In this study, the surface roughness in WAAM is quantitatively determined through optical measurement, which is a non-invasive method and can provide abundant information. In Ref. [7], an optical system was employed to calculate the root mean square error of height deviation for parts deposited by GMAW-based additive manufacturing. However, the work is not systematic and detailed. This paper aims at establishing an approach for modeling the surface roughness, the reconstruction of which is realized through an optical system consisting of a camera and a diode laser.

\section{Experimental Details}

Figure 1 shows a schematic diagram of WAAM. Gas metal arc was utilized as the heat source. The deposition power supply was a Panasonic YD-500FR. The arc was struck between a consumed wire electrode and the top surface of deposited layers. ER50-6 wire electrode with $1.2 \mathrm{~mm}$ diameter was fed into the molten pool for depositing layers. The shielding gas was a mixture of $\mathrm{Ar}(95 \%)$ and $\mathrm{CO}_{2}(5 \%)$ gas with a flow rate of $18 \mathrm{~L} /$ min. The deposition process was conducted on a substrate made of low carbon steel with dimensions of $250 \mathrm{~mm} \times 75 \mathrm{~mm} \times 10 \mathrm{~mm}$.

The movement of the platform was driven by three stepping motors, respectively. It is able to move in the $Y$-axis and to lift in the $Z$-axis, and it can also rotate around the $Z$-axis. During the deposition process, the GMAW torch was kept stationary, and thin-walled parts were performed by the movement of the platform in the $Y$-axis. After each layer was performed, the platform was lowered by a predefined layer height. It is noted that the

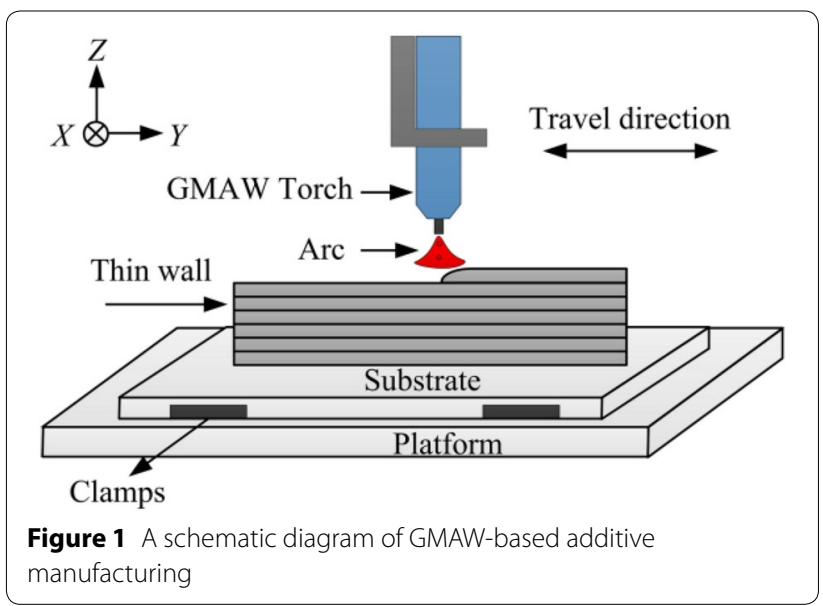

deposition direction between adjacent layers were opposite. This is because the thin-walled part deposited in the same direction presents bad geometries at both ends of the straight wall.

\section{Quantification and Optical Measurement for Surface Roughness}

\subsection{Definition of Surface Roughness}

Figure 2 gives a typical surface profile of the component fabricated by GMAW-based additive manufacturing. As seen in Figure 2, an uneven surface appearance is observed conspicuously in adjacent layers thanks to a layer height more than $1 \mathrm{~mm}$ during multi-layer depositions. This phenomenon is known as the stair stepping effect, which is inevitably produced in additive manufacturing. Due to a larger layer height, this effect is more predominant in WAAM in comparison to that in selective laser melting (SLM). In general, the surface quality of deposited parts has a significant effect on the maching amount of metals during the subsequent finish machining process.

Based on the surface profile given in Figure 2, a schematic diagram shown in Figure 3 can be conducted to present the surface roughness of parts deposited by WAAM. Assuming that points are located on the surface profile in world coordinate system, during multi-layer depositions with a random angle, an idea plane can be calculated by fitting points located on the surface profile through the least square method. The equation of the fitted plane can be written as

$$
A x+B y+C z+D=0,
$$

where $A, B, C, D$ are coefficients of the fitting plane, respectively, $(x, y, z)$ are the variables in the world coordinate system.

Distances between all the surface profile points and the above fitted plane are given by 


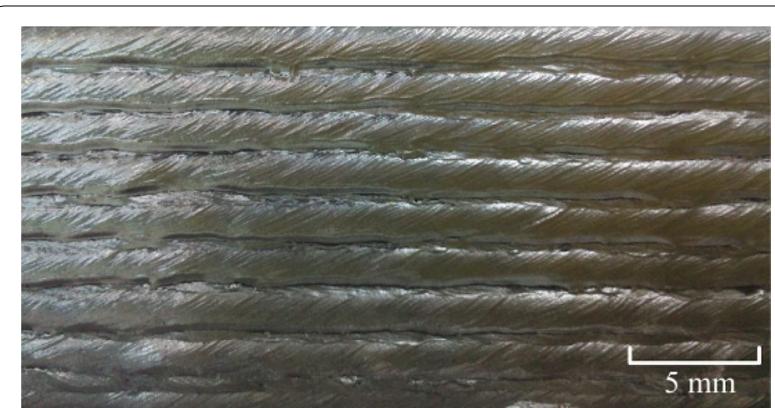

Figure 2 Surface profile of thin-walled part produced in WAAM

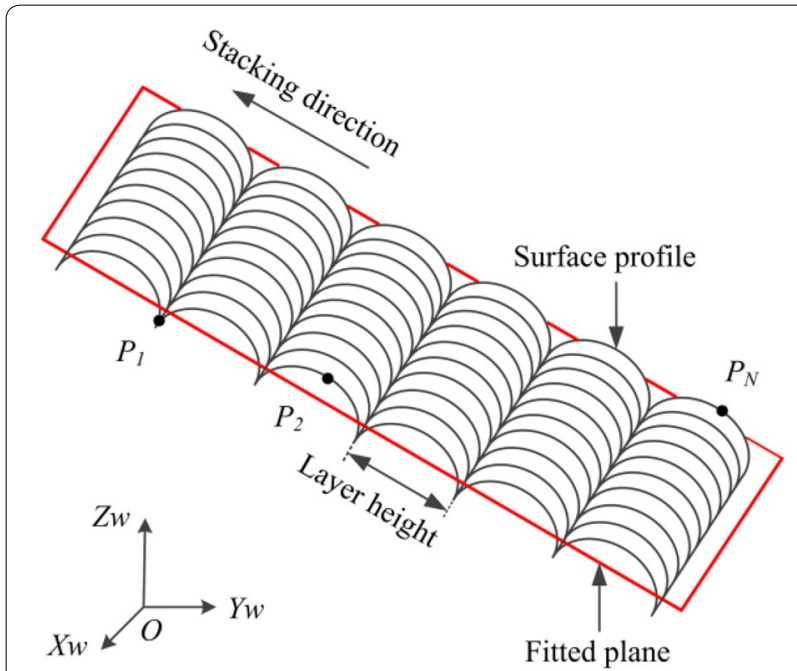

Figure 3 Schematic diagram to quantify average surface roughness of parts

$$
\sum d_{i}=\sum_{i=1}^{N} \frac{\left|A x_{i}+B y_{i}+C z_{i}+D\right|}{\sqrt{A^{2}+B^{2}+C^{2}}},
$$

where $N$ is the total number of fitting points.

Definition of average surface roughness is calculated as

$$
R=\frac{\sum d_{i}}{N}
$$

\subsection{Laser Vision System for Surface Profile}

Based on the definition of surface roughness in the previous section, obtaining the space coordinates of points located on the surface profile is the premise for calculating the surface roughness. Consequently, a key issue is how to calculate the coordinate values. In the present work, the surface profile of the part deposited in WAAM is reconstructed by means of a laser vision sensing system.

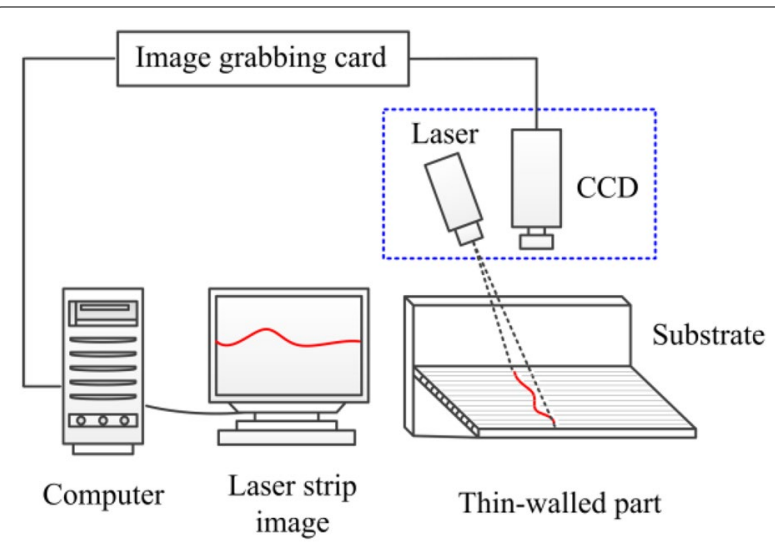

Figure 4 Schematic representation of surface profile measurement

The schematic representation of optical measurement process for the surface profile is displayed in Figure 4. The laser vision sensing system was mounted behind the GMAW torch. The thin-walled part was placed on the platform, and the surface profile was scanned by the laser vision system through the movement of the platform in the $Y$-axis. To reduce noises, the scanning area was away from both ends of the thin-walled part.

The laser sensing system was composed of a charge coupled device (CCD) camera, a diode laser, a neutral and a narrow-band filter. The central wavelength of the narrow-band filter was $650 \mathrm{~nm}$ corresponding to that of the diode laser. This helps the laser light pass through the narrow-band filter and reduces the possibility of other lights passing through the CCD camera. The laser strip was projected to the surface profile of the thin-walled part, and it was captured by the CCD camera. Laser strip images with $400 \times 300$ pixels were transmitted to the computer by the image grabbing card.

During the scanning process for the surface profile, the surface shape variation, surface quality, and noises can affect the extraction accuracy of central line of the laser strip. Consequently, corresponding algorithms, such as Gaussian filter for removing noises and center of gravity method for extracting central line, were applied to treat the laser stripe image. Figure 5 presents a typical image after applying the image process algorithms. To reduce the image processing time, only part of the interested area in the red box was treated, as seen in Figure 5.

\section{Calibration of Laser Vision System}

In the previous section, the laser trip is presented in the two-dimensional image. To quantitatively represent the surface roughness of parts fabricated in WAAM, the laser strip in the image has to be transformed into the world coordinate system. Therefore, the laser vision system is 


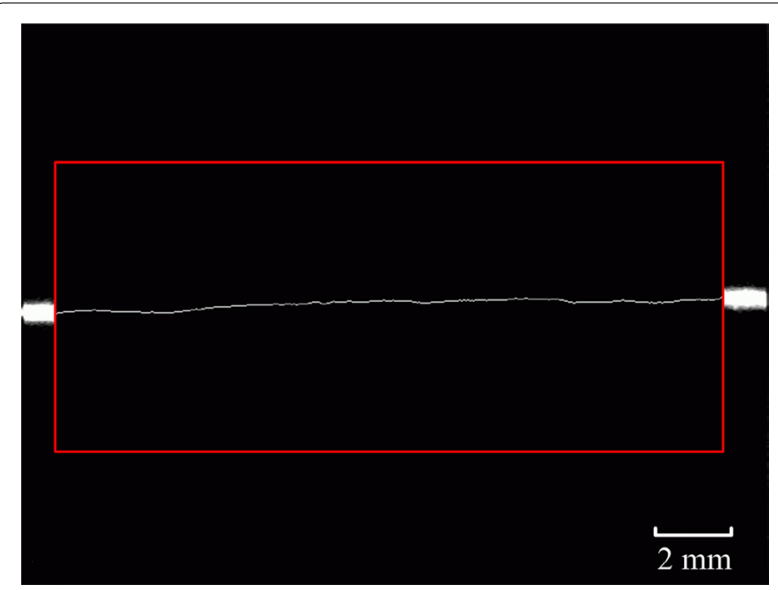

Figure 5 Extraction of laser center line by applying image processing algorithms

necessary to be calibrated. Its calibration which is a onego task is composed of internal and external parameters of the CCD camera as well as the laser plane projected by the diode laser.

For the calibration of the CCD camera, the relationship between a point in the image and in the world coordinate system is given by

$$
s\left[\begin{array}{c}
u \\
v \\
1
\end{array}\right]=\left[\begin{array}{ccc}
\alpha & \gamma & u_{0} \\
0 & \beta & v_{0} \\
0 & 0 & 1
\end{array}\right]\left[\begin{array}{ll}
\boldsymbol{R} & \boldsymbol{T}
\end{array}\right]\left[\begin{array}{c}
X_{w} \\
Y_{w} \\
Z_{w} \\
1
\end{array}\right]
$$

where $(u, v)$ are the image coordinates, $\left(X_{\mathrm{w}}, Y_{\mathrm{w}}, Z_{\mathrm{w}}\right)$ are the world coordinates, $s$ is an arbitrary scale factor, $\boldsymbol{R}$ and $\boldsymbol{T}$ are the external parameters representing rotation matrix and translation vector, respectively, $\left(u_{0}, v_{0}\right)$ are the coordinates of the principal point, $\alpha$ and $\beta$ are the scale factors in image $u$ and $v$ axis, and $\gamma$ is the parameter denoting the skewness of both image axis.

For the calibration of cameras only considering the radial distortion, commonly utilized methods are detailed in Refs. [23, 24]. This approach is easy to be realized through a two-dimensional template without a complicated 3D plane. As a consequence, a two-dimensional template, as presented in Figure 6, was used to calibrate the internal and external parameters of the camera. Each grid in Figure 6 is $1 \times 1 \mathrm{~mm}^{2}$. The template was placed at different angles in the filed range of the camera. Template image at each angle was captured. Twenty images were used as the calibration images. Through approach described in Ref. [22], the calibration results are obtained, namely, focal length $[2477.9,2489.5]$, coordinates of the principal point [173.84, 279.53], distortion coefficient $[-0.7469,1.1106]$.

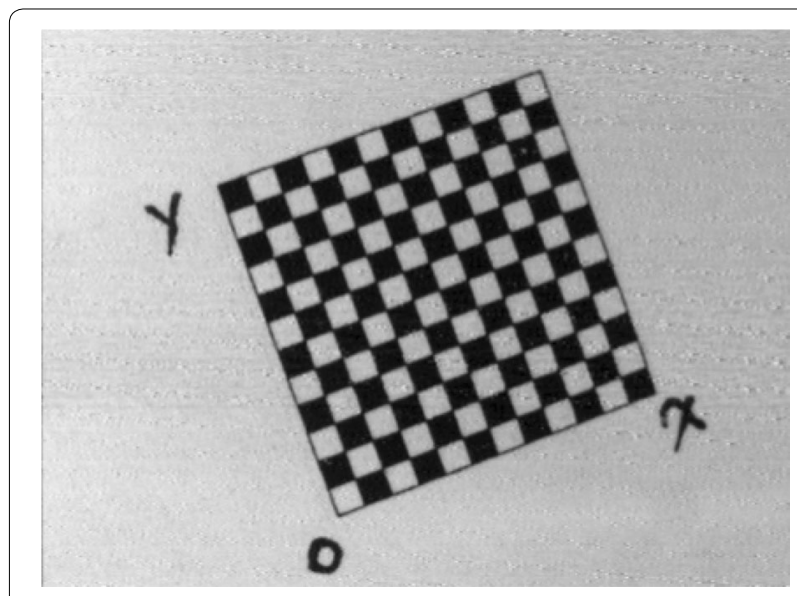

Figure 6 Two-dimensional calibration template

In order to calculate the point coordinate in the world coordinate system, the laser plane projected by the diode laser is also essential to be calibrated. The calibration of the laser plane needs to acquire the position relation between the laser plane and the camera. Supposing that the laser plane in the camera coordinate system $\left(X_{\mathrm{c}}, Y_{\mathrm{c}}\right.$, $Z_{\mathrm{c}}$ ) can be written as

$$
Z_{c}=D X_{c}+E Y_{c}+F
$$

where $D, E, F$ are coefficients of the laser plane, respectively.

During the process of collecting calibration images at different angles for internal and external parameters calibration, the laser strip was also projected on the twodimensional plane. Thus, twenty images with the laser strip were also captured. Although the obtained images were located in different angles, the position of the laser plane was unchanged. Thus, the twenty laser strips should locate in the same plane, which is the laser plane in the camera coordinate system.

Assuming that $Z_{\mathrm{w}}=0$, Eq. (4) can be simplified as

$$
\left[\begin{array}{c}
X_{w} \\
Y_{w} \\
1
\end{array}\right]=s\left[\begin{array}{lll}
r_{1} & r_{2} & \boldsymbol{T}
\end{array}\right]^{-1}\left[\begin{array}{ccc}
\alpha & \gamma & u_{0} \\
0 & \beta & v_{0} \\
0 & 0 & 1
\end{array}\right]^{-1},
$$

where $r_{1}$ and $r_{2}$ are column vectors of rotation matrix $\boldsymbol{R}$.

The relationship between the camera coordinate system on the template and the world coordinate system is given as

$$
\left[\begin{array}{c}
X_{c} \\
Y_{c} \\
Z_{c}
\end{array}\right]=\boldsymbol{R}\left[\begin{array}{c}
X_{w} \\
Y_{w} \\
0
\end{array}\right]+\boldsymbol{T}
$$

Combining Eqs. (6) and (7), the points located on the laser strip in the camera coordinate system can be 


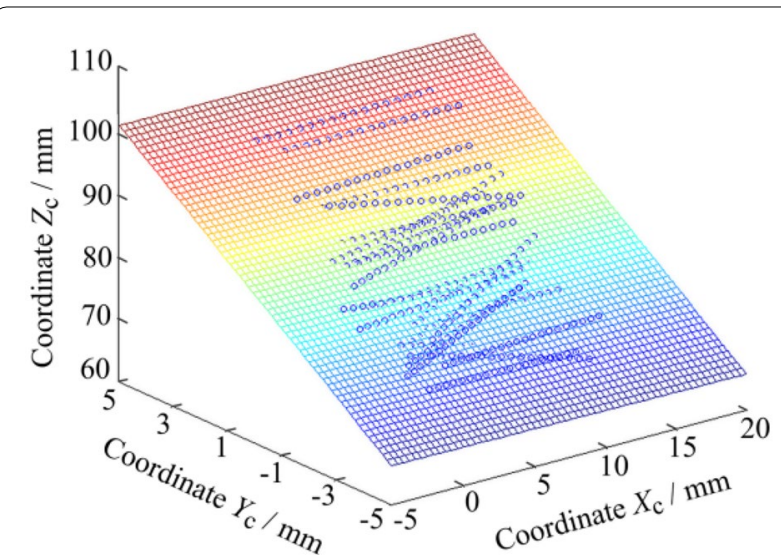

Figure 7 Fitting of laser plane

reconstructed. The fitting of laser plane is shown in Figure 7.

To validate the calibration accuracy of the laser vision sensor, a workpiece with a series of V-type grooves is utilized. As seen in Figure 8(a), the laser strip was projected on the V-type groove located at the right side. Through the calibration of laser plane and internal as well as external parameters of the camera, the surface profile of the V-type groove was reconstructed, as shown in Figure 8(b). The vertical depth between the real and calculated values is compared, and most of absolute errors approach to $0 \mathrm{~mm}$. The maximum absolute error is less than $0.15 \mathrm{~mm}$.

Possible sources of errors for calculated value may be resulted from several factors. The model of the camera is assumed as the pin-hole model. Tangential distortion of the lens is not considered, which inevitably affects the measurement accuracy. Furthermore, the surface quality of parts may make the laser strip scatter, resulting in an increased width of the laser strip and affecting the extraction of the laser center line. Errors in image process algorithms, applied to smooth the image and to extract the laser strip center line, inevitably exist.

\section{Determination of Surface Roughness in WAAM}

A 15-layered straight wall with $160 \mathrm{~mm}$ long was deposited in GMAW-based additive manufacturing with an arc current of $150 \mathrm{~A}$ (wire feed speed $3.73 \mathrm{~m} / \mathrm{min}$ ), travel speed of $5 \mathrm{~mm} / \mathrm{s}$, and arc voltage of $22 \mathrm{~V}$. The inter-layer temperature was controlled at $120^{\circ} \mathrm{C}$. This was achieved by measuring the temperature of the point located on the top surface and in the middle of the thin-wall. As the point temperature was cooled down to $120^{\circ} \mathrm{C}$, a new layer was begun to be deposited. The appearance of the straight wall part is shown in Figure 9.

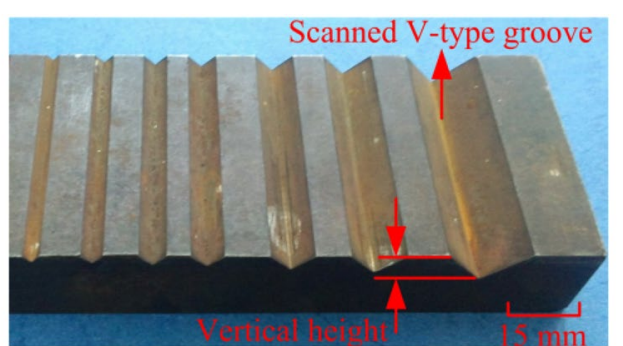

a V-type groove

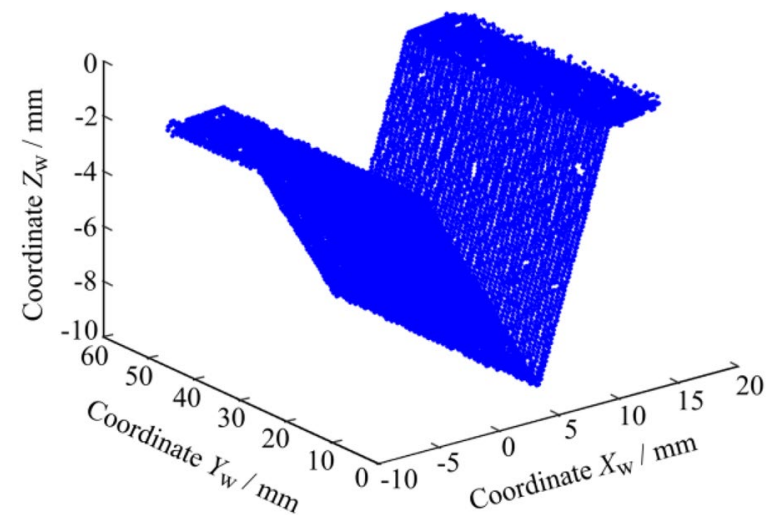

b Reconstruction of surface appearance for V-type groove Figure 8 V-type groove workpiece and its surface reconstruction

The developed laser vision system, fixed on the GMAW gun, was utilized to scan the surface profile of the thinwalled part. The scanning length was approximately $120 \mathrm{~mm}$, and scanning area was away from both ends of the wall. Through the calibration results given in Section 4, the point clouds of the surface profile were reconstructed, as presented in Figure 10. To remove noises, the surface profile of the thin-walled part was scanned for three times. By using Eq. (3), the mean value of the surface roughness was calculated as $0.136 \mathrm{~mm}$. It is seen in Figure 10 that an uneven surface appearance can be observed conspicuously in adjacent layers due to the large layer height induced stair step effect.

The laser vision sensing method proposed to determine the surface roughness of parts fabricated in WAAM is simple and effective. Compared to the commercial laser scanning and the coordinate measurement machine, the equipment cost of laser vision sensing consisting of a cheap CCD and a laser projector is lower, and the volume of the laser vision sensing system is small. Moreover, compared to the cross sectional measurements, the measurement process is easy to realize automation and can treat a number of points quickly. The measurement time of this method mainly depending on the image processing is less than $30 \mathrm{~ms}$. 


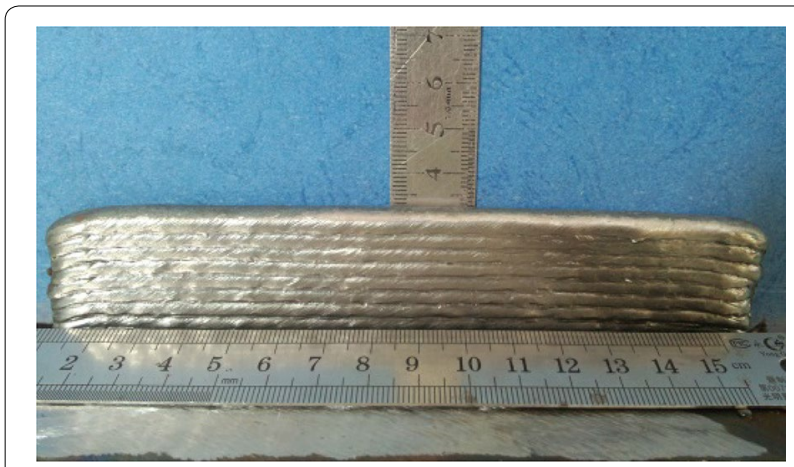

Figure 9 15-layered wall built in WAAM

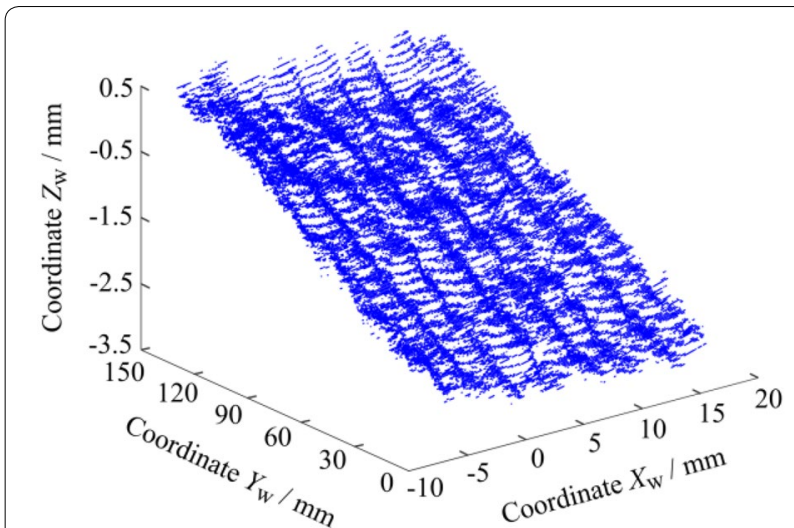

Figure 10 Reconstruction of surface profile for thin-walled part deposited in WAAM

In future, more work will be done to investigate the effect of process parameters, such as the inter-layer temperature, wire feed speed, and travel speed etc., on the surface roughness of deposited parts using the developed method.

\section{Conclusions}

(1) A determination method is proposed to quantify the surface roughness and to reconstruct the surface profile of thin-walled parts deposited in WAAM. The expressions for calculating the surface roughness are presented.

(2) A laser vision system consisting of a diode laser and a CCD camera is utilized to scan the surface profile. By calibrating the camera and the laser plane, points in the world coordinate system are rebuilt from the imaging coordinate system.

(3) Possible sources of error for the reconstruction accuracy are addressed, including the tangential distortion of the lens and uncertainties in image process algorithms. It is demonstrated that the laser vision sensing method is an effective and low-cost method to characterize the surface roughness of parts deposited by WAAM.

\section{Authors' Contributions}

JX was in charge of the whole trial; JX wrote the manuscript; Y-JL, Z-QY and $\mathrm{HC}$ assisted with sampling and laboratory analyses. All authors read and approved the final manuscript.

\section{Authors' Information}

Jun Xiong, born in 1986, is currently an associate professor at School of Materials Science and Engineering, Southwest Jiaotong University, China. He received his PhD degree from Harbin Institute of Technology, China, in 2014. His research interests include wire and arc additive manufacturing.

Yan-Jiang Li, born in 1995, is currently a master candidate at School of Materials Science and Engineering, Southwest Jiaotong University, China. His research interests include sensing and control in additive manufacturing.

Zi-Qiu Yin, born in 1993, is currently a master candidate at School of Materials Science and Engineering, Southwest Jiaotong University, China.

Hui Chen, born in 1970, is currently a professor at Southwest Jiaotong University, China. He received his PhD degree from Sichuan University, China, in 2008. His research interests include laser welding

\section{Competing Interests}

The authors declare no competing financial interests.

\section{Funding}

Supported by National Natural Science Foundation of China (Grant Nos. 51505394, 61573293), and Key Technologies R \& D Program of Sichuan Province of China (Grant No. 2015GZ0305).

\section{Publisher's Note}

Springer Nature remains neutral with regard to jurisdictional claims in published maps and institutional affiliations.

Received: 13 June 2016 Accepted: 10 August 2018

Published online: 23 August 2018

\section{References}

[1] B Baufeld, OV D Biest, R Gault. Additive manufacturing of Ti-6Al-4V components by shaped metal deposition: microstructure and mechanical properties. Materials and Design, 2010, 31: S106-S111.

[2] F Martina, J Mehnen, S W Williams, et al. Investigation of the benefits of plasma deposition for the additive layer manufacture of Ti-6Al-4V. Journal of Materials Processing Technology, 2012, 212: 1377-1386.

[3] H J Wang, W H Jiang, J H Ouyang, et al. Rapid prototyping of 4043 al-alloy parts by VP-GTAW. Journal of Materials Processing Technology, 2004, 148: 93-102.

[4] F D Wang, S Williams, M Rush. Morphology investigation on direct current pulsed gas tungsten arc welded additive layer manufactured Ti6Al4V alloy. The International Journal of Advanced Manufacturing Technology, 2011, 57: 597-603.

[5] XH Xiong. A new method of direct metal prototyping: hybrid plasma deposition and milling. Rapid Prototyping Journal, 2008, 14(1): 53-56.

[6] S Jhavar, N K Jain, C P Paul. Development of micro-plasma transferred arc ( $\mu$-PTA) wire deposition process for additive layer manufacturing applications. Journal of Materials Processing Technology, 2014, 214(5): 1102-1110.

[7] J D Spencer, P M Dickens, C M Wykes. Rapid prototyping of metal parts by three-dimensional welding. Proceedings of the Institution of Mechanical Engineers Part B Journal of Engineering Manufacture, 1998, 212: 175-182.

[8] Y M Zhang, Y W Chen, P J Li, et al. Weld deposition-based rapid prototyping: a preliminary study. Journal of Materials Processing Technology, 2003, 135: 347-357.

[9] Y A Song, S Park. Experimental investigations into rapid prototyping of composites by novel hybrid deposition process. Journal of Materials Processing Technology, 2006, 171: 35-40. 
[10] J Xiong, G J Zhang, H M Gao, et al. Modeling of bead section profile and overlapping beads with experimental validation for robotic GMAW-based rapid manufacturing. Robotics and Computer-Integrated Manufacturing, 2013, 29(2): 417-423.

[11] S Suryakumar, K P Karunakaran, A Bernard, et al. Weld bead modeling and process optimization in hybrid layered manufacturing. Robotics and Computer-Integrated Manufacturing, 2011, 43(4): 331-344.

[12] P Kazanas, P Deherkar, P Almeida, et al. Fabrication of geometrical features using wire and arc additive manufacture. Proceedings of the Institution of Mechanical Engineers Part B Journal of Engineering Manufacture, 2012, 226(B6): 1042-1051.

[13] A Heralic, A K Christiansson, B Lennartson, et al. Height control of laser metal-wire deposition based on iterative learning control and 3D scanning. Optics and Lasers in Engineering, 2012, 50: 1230-1241.

[14] J Xiong, G J Zhang. Online measurement of bead geometry in GMAWbased additive manufacturing using passive vision. Measurement Science and Technology, 2013, 24(11): 115103.

[15] J Xiong, G J Zhang. Adaptive control of deposited height in GMAW-based layer additive manufacturing. Journal of Materials Processing Technology, 2014, 214: 962-968

[16] H H Zhao, G J Zhang, Z Q Yin, et al. A 3D dynamic analysis of thermal behavior during single-pass multi-layer weld-based rapid prototyping. Journal of Materials Processing Technology, 2011, 211(3): 488-495.

[17] P A Colegrove, H E Coules, J Fairman, et al. Microstructure and residual stress improvement in wire and arc additively manufactured parts through high-pressure rolling. Journal of Materials Processing Technology, 213(10): 1782-1791
[18] K Cheng, N Aris. Characterization of the surface functionality on precision machined engineering surfaces. The International Journal of Advanced Manufacturing Technology, 2008, 38: 402-409.

[19] P M Pandey, N V Reddy, S G Dhande. Improvement of surface finish by staircase machining in fused deposition modeling. Journal of Materials Processing Technology, 2003, 132: 323-331.

[20] D Ahn, H Kim, S Lee. Surface roughness prediction using measured data and interpolation in layered manufacturing. Journal of Materials Processing Technology, 2009, 209(2): 664-671.

[21] B K Paul, V Voorakarnam. Effect of layer thickness and orientation angle on surface roughness in laminated object manufacturing. Journal of Manufacturing Processes, 2001, 3(2): 94-101.

[22] D Ahn, J H Kweon, J Choi, et al. Quantification of surface roughness of parts processed by laminated object manufacturing. Journal of Manufacturing Processes, 2012, 212(2): 339-346.

[23] ZY Zhang. A flexible new technique for camera calibration. IEEE Transactions on Pattern Analysis and Machine Intelligence, 2000, 22(11): 1330-1334

[24] RY Tsai. A versatile camera calibration technique for high-accuracy 3D machine vision metrology using off-the-shelfTV cameras and lenses. IEEE Journal of Robotics and Automation RA, 1987, 3(4): 323-344.

\section{Submit your manuscript to a SpringerOpen ${ }^{\odot}$ journal and benefit from:}

- Convenient online submission

$\checkmark$ Rigorous peer review

- Open access: articles freely available online

- High visibility within the field

- Retaining the copyright to your article

Submit your next manuscript at $\boldsymbol{\nabla}$ springeropen.com 